\title{
Role of the Antiferromagnetic Bulk Spin Structure on Exchange Bias
}

\author{
R. Morales, ${ }^{1,2}$ Zhi-Pan Li, ${ }^{1}$ J. Olamit, ${ }^{3}$ Kai Liu, ${ }^{3}$ J. M. Alameda, ${ }^{2}$ and Ivan K. Schuller ${ }^{1}$ \\ ${ }^{1}$ Physics Department, University of California-San Diego, La Jolla, California 92093, USA \\ ${ }^{2}$ Departamento de Física, Universidad de Oviedo-CINN, Oviedo, 33007 Spain \\ ${ }^{3}$ Physics Department, University of California, Davis, California 95616, USA \\ (Received 29 December 2007; revised manuscript received 28 October 2008; published 4 March 2009)
}

\begin{abstract}
The cooling field dependence of the exchange bias field in ferromagnet/antiferromagnet (FM/AF) multilayers demonstrates that the bulk AF spin structure plays a crucial role on the origin of exchange bias. FM/AF/FM trilayers were designed to eliminate any interlayer exchange coupling between the FM slabs. By choosing the magnetic cooling field, the AF is ordered below its Néel temperature with the FM layers fully saturated either parallel or antiparallel to each other. The significant difference in the exchange bias field between these two cooling configurations confirms that exchange bias cannot be a purely interfacial effect and that the bulk AF moments play a significant role in pinning the uncompensated spins at the $\mathrm{AF} / \mathrm{FM}$ interface. This experiment also demonstrates that the mechanism responsible for coercivity enhancement has a different origin and is independent of the process that gives rise to exchange bias.
\end{abstract}

DOI: 10.1103/PhysRevLett.102.097201

PACS numbers: 75.70.-i, 75.30.Et, 75.50.Ee, 75.60.-d

Since its discovery [1], exchange bias (EB) has been intensively studied due to its importance in spintronics, especially in spin valve design. However, the elusive EB mechanism is still not well understood [2,3]. The shift of the hysteresis loop $M(H)$ in ferromagnet/antiferromagnet (FM/AF) systems, which defines the exchange bias field $H_{\mathrm{EB}}$, is a consequence of the exchange coupling between $\mathrm{FM}$ and AF spins at the interface [4]. For this reason, most EB models consider only the spin configuration near the interface. For example, domain walls in the AF perpendicular to the FM/AF interface set up the unidirectional anisotropy in Malozemoff's model [5], while spiral-like domain walls parallel and close to the AF/FM interface are proposed in Mauri's model [6]. Other approaches with different spin configurations assume that the AF bulk plays no role in determining the interfacial spin configuration and the magnitude of EB [7,8]. On the contrary, the domain state model [9] considers the entire volume of the AF in order to account for EB, suggesting that the internal magnetic structure of the AF is crucial for the emergence of EB. Several techniques have been applied to study AF domains [10-12], and $90^{\circ}$ or $180^{\circ}$ domain walls were claimed for different systems [13-20]. Although recent experiments [21,22] suggest that the bulk AF sets the $\mathrm{EB}$, whether $\mathrm{EB}$ is purely controlled by the interface or AF bulk is still controversial.

In this Letter, we demonstrate the existence of a bulk AF magnetic structure which plays an important role in pinning the uncompensated interfacial moments that account for exchange bias. The procedure, based upon FM1/AF/ FM2 trilayers, is simple and conclusive. No interlayer exchange coupling exists between the FM1/AF and AF/ FM2 interfaces, and the entire experiment is done while keeping the same morphological properties. This is a new way in which the AF bulk is manipulated with potential for controlling the EB magnitude using the cooling configuration of the trilayer. Our experiment also demonstrates that the mechanism responsible for coercivity enhancement is independent of the origin of EB.

The ideal system for this study is a FM1/AF/FM2 trilayer with decoupled FMs, square hysteresis loops above the Néel temperature of the AF, $T_{N}$, and well-separated coercivities. In this way, the system can be cooled down below $T_{N}$ with FM1 and FM2 magnetizations saturated parallel or antiparallel to each other by a judicious choice of cooling field $H_{\mathrm{FC}}$. Positive $H_{\mathrm{FC}}$ saturates both FMs in the same direction and freezes the AF in the so-called "parallel configuration," whereas negative $H_{\mathrm{FC}}$ between the FM1 and FM2 coercivities reverses only the soft FM magnetization and arranges the $\mathrm{AF}$ spins below $T_{N}$ in the "antiparallel configuration" (insets in Fig. 1). If EB is a purely interfacial effect, then the $H_{\mathrm{EB}}$ of each FM should be independent of the cooling configuration; i.e., it should have the same absolute value for parallel or antiparallel configurations. In contrast, if the bulk AF structure couples the uncompensated spins of FM1/AF and AF/FM2 interfaces during the cooling process, $H_{\mathrm{EB}}$ may depend on the cooling configuration.

$\mathrm{Ni}(50 \mathrm{~nm}) / \mathrm{FeF}_{2}\left(t_{\mathrm{AF}}\right) / \mathrm{Py}(50 \mathrm{~nm}) \quad[\mathrm{FM} 1=\mathrm{Ni}, \mathrm{FM} 2=$ Permalloy (Py)] trilayers were deposited by electron beam evaporation on $\mathrm{MgF}_{2}$ (110) single crystal substrate. Samples were protected from oxidation by an $\mathrm{Al}(4 \mathrm{~nm})$ film. The AF thickness $t_{\mathrm{AF}}$ varies from 2 to $200 \mathrm{~nm}$. Magnetic characterization was performed by vibrating sample magnetometry (VSM) and the magneto-optical Kerr effect (MOKE). VSM measures the full magnetic moment of the sample, while the separate magnetization of each FM layer is determined from the MOKE by illuminating the sample from the top or bottom side through the transparent substrate. The penetration depth of the 


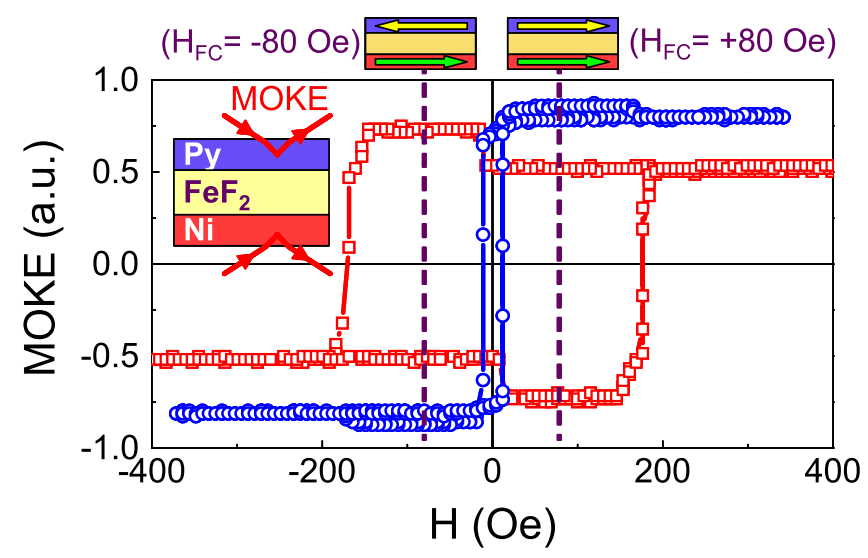

FIG. 1 (color online). MOKE room temperature hysteresis loops from $\mathrm{Ni}$ and Py layers in $\mathrm{Ni}(50 \mathrm{~nm}) / \mathrm{FeF}_{2}(200 \mathrm{~nm}) /$ Py $(50 \mathrm{~nm})$ trilayers. Insets: MOKE probing each FM individual layer and $\mathrm{Ni}$ and Py magnetization alignment for $H_{\mathrm{FC}}=+80$ and $-80 \mathrm{Oe}$ (parallel and antiparallel cooling configuration, respectively).

light, less than $50 \mathrm{~nm}$ for the FM layer, enables us to obtain accurately the EB field $H_{\mathrm{EB}}$ and coercivity $H_{C}$ of each individual layer.

The cooling configuration is set above the Néel temperature of $\mathrm{FeF}_{2}\left(T_{N}=78 \mathrm{~K}\right) . H_{\mathrm{FC}}$ arranges the $\mathrm{Ni}$ and Py layers in either the parallel or antiparallel configuration along the easy axis of the films. Then the trilayer is cooled below $T_{N}$, and the AF spins are ordered in the parallel or antiparallel configuration.

Samples with $t_{\mathrm{AF}}<10 \mathrm{~nm}$ show an interlayer exchange coupling between the two FMs above $T_{N}$ and thus are excluded from our studies. However, at $t_{\mathrm{AF}}>20 \mathrm{~nm}$ no signature of such coupling exists, and thus these are ideal for this study. Here we will focus on the sample with $t_{\mathrm{AF}}=$ $200 \mathrm{~nm} \quad\left[\mathrm{Ni}(50 \mathrm{~nm}) / \mathrm{FeF}_{2}(200 \mathrm{~nm}) / \mathrm{Py}(50 \mathrm{~nm})\right]$. The same behavior exhibited by this trilayer was also observed in all other samples with $20 \mathrm{~nm}<t_{\mathrm{AF}}<250 \mathrm{~nm}$, but the quantitative $H_{\mathrm{EB}}$ change upon parallel and antiparallel cooling was different.

Figure 1 shows MOKE hysteresis loops from the individual Ni and Py layers at room temperature. The squareness of the loops and the well-separated coercivities make this an ideal system for this study [23]. The sample is first fully saturated along the positive direction and the field reduced to $H_{\mathrm{FC}}$. For $H_{\mathrm{FC}}=+80 \mathrm{Oe}$, both ferromagnets are saturated with parallel alignment setting up the parallel configuration. At $H_{\mathrm{FC}}=-80 \mathrm{Oe}$, only the Py magnetization has reversed, and thus the system is cooled into the antiparallel configuration. The square Ni and Py hysteresis loops allow the comparison between the two procedures since both ferromagnets are saturated in each cooling configuration.

MOKE hysteresis loops were measured at different temperatures for each FM layer. To compare the EB from both cooling states, the absolute value of $H_{\mathrm{EB}}$ versus $T$ is plotted

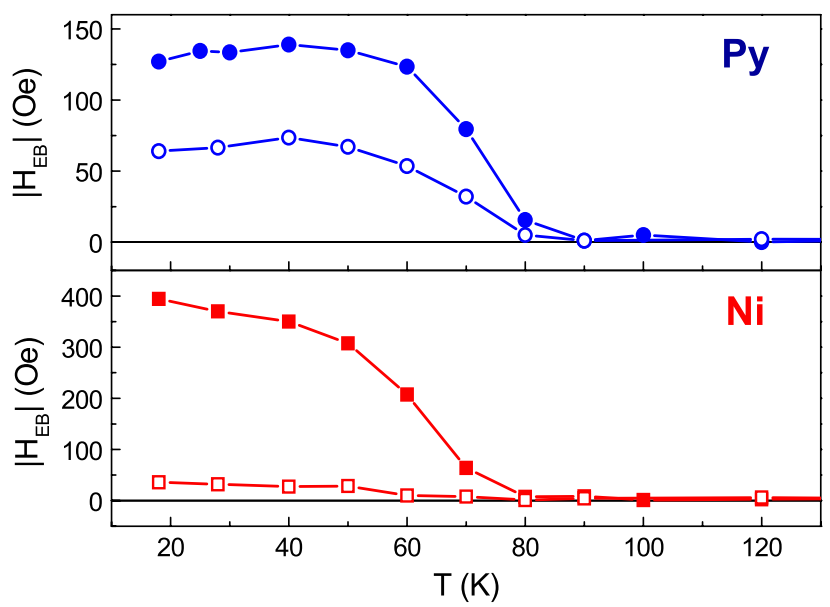

FIG. 2 (color online). Dependence of the Ni and Py exchange bias field for both parallel (solid symbols) and antiparallel (open symbols) cooling configurations.

in Fig. 2. The highest values correspond to the parallel cooling configuration with $H_{\mathrm{EB}} \approx 130$ Oe for Py and $400 \mathrm{Oe}$ for $\mathrm{Ni}$ at low temperature. However, these values change significantly in the antiparallel configuration. $H_{\mathrm{EB}} \approx 65 \mathrm{Oe}$ for $\mathrm{Py}$ and $40 \mathrm{Oe}$ for $\mathrm{Ni}$; i.e., the $\mathrm{EB}$ is reduced by a factor of 2 for Py and one order of magnitude for Ni. This drastic decrease in the EB magnitude demonstrates that there must be an internal magnetic structure in the bulk of the AF, which couples the uncompensated spins of both $\mathrm{Ni} / \mathrm{FeF}_{2}$ and $\mathrm{FeF}_{2} /$ Py interfaces during the cooling process. We have verified that this remarkable reduction of $H_{\mathrm{EB}}$ is not intrinsic to the cooling process of separate interfaces. $\mathrm{Ni} / \mathrm{FeF}_{2}$ bilayers were prepared with the same layer thicknesses and crystallinity as the trilayers. The bilayer was positively saturated, and then the field was reduced to either a positive or negative cooling field, smaller than Ni coercivity. No change of $H_{\mathrm{EB}}$ was observed. This confirms that the EB reduction in the Ni layer, upon cooling the trilayer in a negative field, is a consequence and an effect of the $\mathrm{FeF}_{2} / \mathrm{Py}$ interface through the thickness of the AF.

The remarkable change in $H_{\mathrm{EB}}$ cannot be explained if EB is a purely interfacial effect, and therefore the bulk structure of the AF must be involved. Since the exchange bias is set ultimately by the uncompensated pinned interfacial spins, our results imply that these are determined by interactions with the bulk AF spins. During the cooling process, there is no magnetic coupling between $\mathrm{Ni} / \mathrm{FeF}_{2}$ and $\mathrm{FeF}_{2} / \mathrm{Py}$ interfaces, and the bulk AF spin structure is established by the relative alignment of AF spins near the two interfaces. These AF spins are polarized by the nearby FM. When the system is cooled with parallel FM magnetizations, AF domains extend from both interfaces creating an AF magnetic structure that yields the maximum EB. On the contrary, in the antiparallel cooling process the interfacial AF spins couple to $\mathrm{Ni}$ and Py magnetic moments, 
creating opposite AF domains in the bulk of the AF layer. If the AF bulk plays no role on $\mathrm{EB}$, this opposite alignment should have no effect on the $H_{\mathrm{EB}}$ of each individual FM. However, the formation of the opposite domains in the AF leads to the formation of domain walls in the bulk. The spin structure within the $\mathrm{AF}$ is arranged so as to minimize the energy of a configuration with opposite interfacial domains. The result is a reduction in the net number of uncompensated spins coupled to the FM and therefore a decrease of $H_{\mathrm{EB}}$. In short, the bulk AF plays a crucial role in pinning the interfacial uncompensated spins which give rise to EB.

The difference in the magnetic structure in the $\mathrm{AF}$ between the two cooling configurations is revealed not only in the $H_{\mathrm{EB}}$ magnitude but also in the shape of the hysteresis loops. Low temperature hysteresis loops are more sheared in the antiparallel and sharper in the parallel configuration (Fig. 3). This is also observed in first-order reversal curves $[24,25]$ that show a much broader distribution of local coercivity and bias in the antiparallel configuration. This effect could be attributed to either a different $\mathrm{AF}$ spin structure or a change in the reversal mechanism due to the $H_{\mathrm{EB}}$ decrease (for instance, the change in $\mathrm{Ni}$ at $18 \mathrm{~K}$ from $H_{\mathrm{EB}}=400$ Oe in the parallel to 40 Oe in the antiparallel configuration). The latter was rejected by comparing Ni hysteresis loops for the two cooling configurations with the same $H_{\mathrm{EB}}$, e.g., $\mathrm{Ni} H_{\mathrm{EB}}=40 \mathrm{Oe}$ at $18 \mathrm{~K}$ in the antiparallel configuration and a similar value of $H_{\mathrm{EB}}$ at $73 \mathrm{~K}$ in the parallel one (Fig. 2). VSM hysteresis loops at $18 \mathrm{~K}$ (antiparallel) and $73 \mathrm{~K}$ (parallel) are shown in Fig. 3. The Ni reversal at $73 \mathrm{~K}$ after cooling in the parallel configuration is abrupt, while that after the antiparallel cooling configuration shows a gradual magnetization reversal. Thus, the difference in hysteresis loop shapes is not due to a different exchange coupling strength. Furthermore, the hysteresis loop squareness (shear) produced by the parallel (antiparallel) cooling is maintained for all

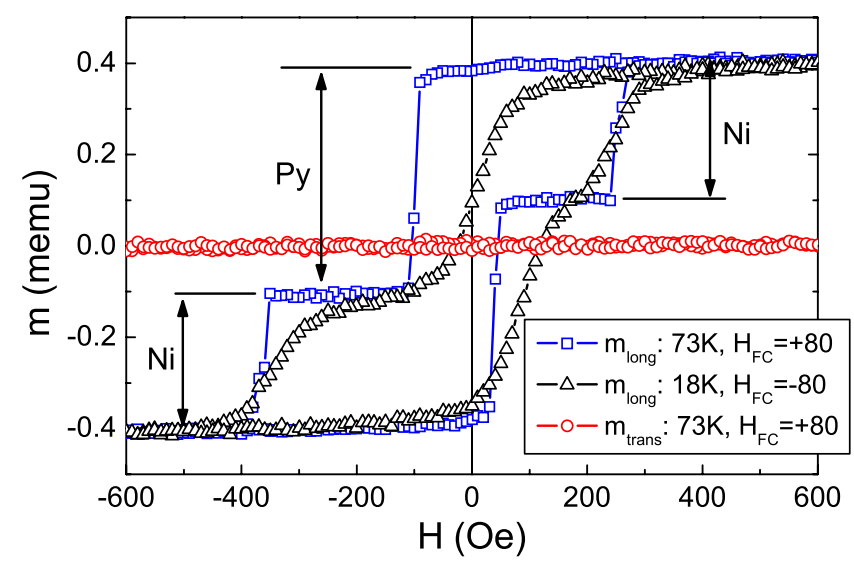

FIG. 3 (color online). VSM longitudinal and transverse hysteresis loops with the same $H_{\mathrm{EB}}$ of $\mathrm{Ni}$. Loop at $18 \mathrm{~K}$ for antiparallel cooling $\left(H_{\mathrm{FC}}=-80 \mathrm{Oe}\right)$ and $73 \mathrm{~K}$ for parallel cooling $\left(H_{\mathrm{FC}}=+80 \mathrm{Oe}\right)$. temperatures below $T_{N}$. Therefore, the origin of the different hysteresis loop shapes is attributed to the AF spin structure created during the cooling process.

Recently, Yang and Chien proposed the formation of spiral domain walls in a Py/FeMn/Co trilayer for $t_{\mathrm{AF}}<$ $15 \mathrm{~nm}$ according to Mauri's model [18]. This structure is possible in $15 \mathrm{~nm}$ thick FeMn which has a domain wall width $\delta_{w} \approx 20 \mathrm{~nm}$, i.e., $t_{\mathrm{AF}} \approx \delta_{w}$. However, in our case $t_{\mathrm{AF}} \gg \delta_{w}$, because $\delta_{w}$ is only a few monolayers due to the strong $\mathrm{FeF}_{2}$ uniaxial magnetic anisotropy [26]. A springlike domain wall in our $\mathrm{AF}$ layer $\left(\mathrm{FeF}_{2} 200 \mathrm{~nm}\right)$ is not probable. Moreover, the reversal of the interfacial AF layer in Mauri's model would produce FM moment rotation, and this FM inversion mode has not been observed in our samples as described below. The transverse magnetization component, perpendicular to the applied field in the sample plane, was measured by VSM for both cooling procedures (Fig. 3). The transverse moment is always null during reversal, indicating that there is no net magnetic moment perpendicular to the external field (Fig. 3 shows only the transverse loops for one cooling configuration; the transverse component of the other one is also null).

Our experiment demonstrates the crucial role of the AF bulk, but Mauri's assumptions cannot explain the results. A possible explanation could be found in the domain state model that attributes EB to the formation of domains throughout the AF volume [9].

The typical coercive field $H_{C}$ enhancement is found in the temperature dependence for Ni and Py layers (Fig. 4). This enhancement has recently been investigated since it is believed to be intimately related to the microscopic origin of EB [7,27-29]. However, our results demonstrate that there is no direct correlation between $H_{\mathrm{EB}}$ and $H_{C}$ [30]. The $H_{C}$ temperature dependence is exactly the same for the parallel and antiparallel cooling configurations. Even an order of magnitude change in $H_{\mathrm{EB}}$, as found for $\mathrm{Ni}$, has no

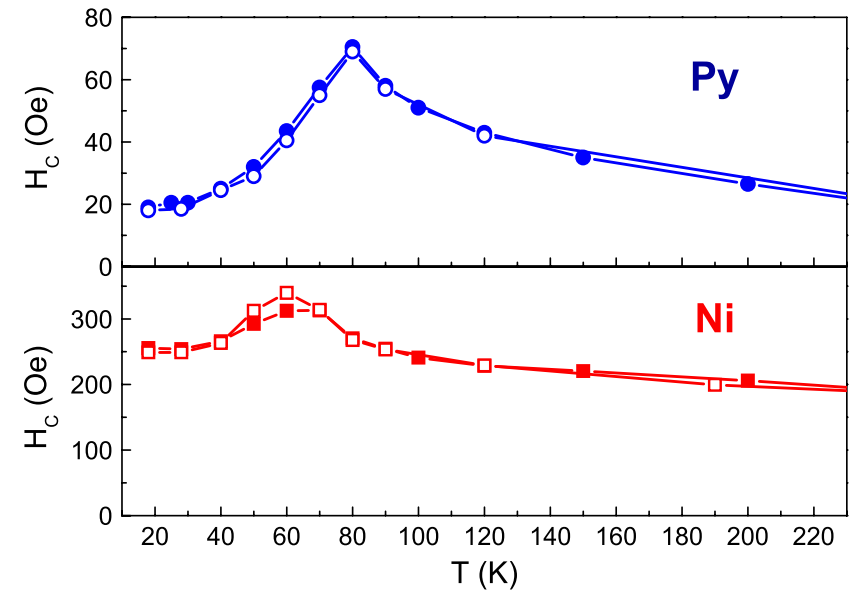

FIG. 4 (color online). Dependence of $\mathrm{Ni}$ and Py coercivity with temperature for both parallel (solid symbols) and antiparallel (open symbols) cooling configurations. 
effect on the temperature evolution of coercivity. This implies that the mechanism responsible for coercivity enhancement must be decoupled and independent of the mechanism that gives rise to EB.

In conclusion, we have shown that the magnitude of pinned uncompensated interfacial AF moments that give rise to $\mathrm{EB}$ depends on not only the interfacial spins but also the entire bulk AF magnetic structure. This conclusive experiment allow us to control the EB magnitude by changing only the cooling conditions and not the system morphology, which could otherwise alter the interface and lead to inconclusive results about the origin of EB.

We gratefully acknowledge enlightening discussions with M. Fitzsimmons (LANL). Work at UCSD is supported by the U.S. Department of Energy and European MarieCurie OIF (R. M.). Work at UCD is supported by ACS-PRF (No. 43637-AC10), CITRIS, and Alfred P. Sloan Foundation. R. M. and J. M. A also acknowledge Spanish MEC FIS2005-07392 and FIS2008-06249 funding.

[1] W. H. Meiklejohn and C.P. Bean, Phys. Rev. 102, 1413 (1956).

[2] J. Nogués and Ivan K. Schuller, J. Magn. Magn. Mater. 192, 203 (1999).

[3] M. Kiwi, J. Magn. Magn. Mater. 234, 584 (2001).

[4] W. H. Meiklejohn, J. Appl. Phys. 33, 1328 (1962).

[5] A. P. Malozemoff, Phys. Rev. B 35, 3679 (1987).

[6] D. Mauri, H.C. Siegmann, P. S. Bagus, and E. Kay, J. Appl. Phys. 62, 3047 (1987).

[7] T. C. Schulthess and W. H. Butler, Phys. Rev. Lett. 81, 4516 (1998).

[8] M. Kiwi, J. Mejía-López, R. D. Portugal, and R. Ramírez, Appl. Phys. Lett. 75, 3995 (1999).

[9] U. Nowak, K. D. Usadel, J. Keller, P. Miltényi, B. Beschoten, and G. Güntherodt, Phys. Rev. B 66, 014430 (2002).

[10] F. Nolting, A. Scholl, J. Stöhr, J. W. Seo, J. Fompeyrine, H. Siegwart, J.-P. Locquet, S. Anders, J. Lüning, E. E. Fullerton, M.F. Toney, M.R. Scheinfein, and H.A. Padmore, Nature (London) 405, 767 (2000).

[11] A. Scholl, J. Stöhr, J. Lüning, J. W. Seo, J. Fompeyrine, H. Siegwart, J.-P. Locquet, F. Nolting, S. Anders, E. E. Fullerton, M. R. Scheinfein, and H. A. Padmore, Science 287, 1014 (2000).

[12] C. L. Chien, V. S. Gornakov, V. I. Nikitenko, A. J. Shapiro, and R. D. Shull, Phys. Rev. B 68, 014418 (2003).

[13] J. Camarero, Y. Pennec, J. Vogel, M. Bonfim, S. Pizzini, F. Ernult, F. Fettar, F. Garcia, F. Lancon, L. Billard, B. Dieny,
A. Tagliaferri, and N.B. Brookes, Phys. Rev. Lett. 91, 027201 (2003).

[14] M. H. Pan, J. Chen, J. G. Long, L. N. Tong, M. Lu, J. Du, A. Hu, and H. R. Zhai, J. Magn. Magn. Mater. 226-230, 1817 (2001).

[15] C. Schanzer, V. R. Shah, T. Gutberlet, M. Gupta, P. Böni, and H.B. Braun, Physica (Amsterdam) 356B, 46 (2005).

[16] P. A. A. van der Heijden, C. H. W. Swüste, W. J. M. de Jonge, J. M. Gaines, J. T. W. M. van Eemeren, and K. M. Schep, Phys. Rev. Lett. 82, 1020 (1999).

[17] V. K. Sankaranarayanan, S. M. Yoon, D. Y. Kim, C. O. Kim, and C. G. Kim, J. Appl. Phys. 96, 7428 (2004).

[18] F. Y. Yang and C.L. Chien, Phys. Rev. Lett. 85, 2597 (2000).

[19] G. Malinowski, M. Hehn. S. Robert, O. Lenoble, and A. Schuhl, Phys. Rev. B 68, 184404 (2003).

[20] P. Steadman, M. Ali, A. T. Hindmarch, C. H. Marrows, B. J. Hickey, S. Langridge, R. M. Dalgliesh, and S. Foster, Phys. Rev. Lett. 89, 077201 (2002).

[21] C. W. Leung and M. G. Blamire, Phys. Rev. B 72, 054429 (2005).

[22] P. Miltényi, M. Gierlings, J. Keller, B. Beschoten, G. Güntherodt, U. Nowak, and K. D. Usadel, Phys. Rev. Lett. 84, 4224 (2000).

[23] The small jump in the Ni loop just at the Py coercivity corresponds to a magneto-optical artifact. The light penetrates the whole Ni layer, goes through the transparent $\mathrm{FeF}_{2}$, and probes a very thin slab of Py. The magnetooptical signal from this slab has the opposite sign as has been demonstrated in very thin films [C. Dehesa-Martínez, L. Blanco-Gutierrez, M. Vélez, J. Díaz, L. M. AlvarezPrado, and J.M. Alameda, Phys. Rev. B 64, 024417 (2001)]. The same artifact is observed in Py loops just at the Ni coercivity.

[24] J.E. Davies, O. Hellwig, E. E. Fullerton, G. Denbeaux, J. B. Kortright, and K. Liu, Phys. Rev. B 70, 224434 (2004).

[25] J. Olamit, K. Liu, Z. P. Li, and I. K. Schuller, Appl. Phys. Lett. 90, 032510 (2007).

[26] D. Lederman, R. Ramírez, and M. Kiwi, Phys. Rev. B 70, 184422 (2004).

[27] C. Leighton, J. Nogués, B. J. Jönsson-Akerman, and I. K. Schuller, Phys. Rev. Lett. 84, 3466 (2000).

[28] M.D. Stiles and R.D. McMichael, Phys. Rev. B 63, 064405 (2001).

[29] Y. J. Tang, B. Roos, T. Mewes, S. O. Demokritov, B. Hillebrands, and Y.J. Wang, Appl. Phys. Lett. 75, 707 (1999).

[30] L. E. Fernandez-Outon and K. O'Grady, J. Magn. Magn. Mater. 290, 536 (2005). 